\title{
Avaliação da padronização de agulhas anestésicas odontológicas comercializadas no Brasil
}

\section{Evaluation of the standardization of injection dental needles marketed in Brazil}

\author{
Marcos Antonio GIROTTO* \\ Maria Cristina VOLPATO** \\ José RANALI***
}

\begin{abstract}
GIROTTO, M. A.; VOLPATO, M. C.; RANALI, J. Avaliação da padronização de agulhas anestésicas odontológicas comercializadas no Brasil. Pesqui Odontol Bras, v. 14, n. 3, p. 294-299, jul./set. 2000.

Foram avaliadas neste trabalho agulhas anestésicas odontológicas descartáveis, comercializadas no Brasil no segundo semestre de 1997, quanto às medidas: comprimento da cânula, da porção que perfura o tubete e da porção que permanece no interior do adaptador plástico e diâmetros externo e interno. A resistência à corrosão da cânula e as informações da embalagem (caixa e protetor) também foram avaliadas. Os resultados foram comparados com as normas Internacional (ISO) ${ }^{8}$, Americana (ANSI/ADA) ${ }^{1}$ e Francesa (NF). Foram observadas variações, não havendo uma só marca que estivesse de acordo com qualquer das normas, para todas as medidas. Todas as agulhas atingiram as especificações do teste de corrosão. Algumas embalagens mostraram conteúdo de informação incompleto e uma marca estava sendo vendida sem prazo de validade. Alguns protetores não permitiam identificar a violação do lacre, quando isto ocorria. Estes resultados demonstram a necessidade de se estabelecerem normas brasileiras, para regulamentar a produção e venda de agulhas odontológicas no país.
\end{abstract}

UNITERMOS: Agulhas; Controle de qualidade.

\section{INTRODUÇÃO}

Apesar da importância da agulha na anestesia odontológica, poucos são os trabalhos relatados na literatura, com relação às propriedades essenciais desse produto.

Quando se compara o comprimento e o calibre de agulhas de diferentes marcas comerciais, podem-se obter resultados bizarros, como foi constatado no estudo de WITTROCK; FISCHER ${ }^{14}$ (1968) que encontrou uma marca de agulha $30 \mathrm{G}$ apresentando o mesmo diâmetro de agulhas $25 \mathrm{G}$, de três outras marcas comerciais.

Para evitar esse tipo de aberração, bem como garantir um produto de qualidade aos consumidores, a fabricação de agulhas odontológicas descartáveis passou a ser regulamentada, em vários países, pelo órgão responsável, que estabelece normas de padronização, como é o caso do American National Standard Institute/American Dental Association (ANSI/ADA) ${ }^{1}$, da Internacional Organization for Standardization (ISO) ${ }^{8}$ e da Norma Francesa (NF).
Dada a inexistência de normas brasileiras que regulamentem a produção de agulhas anestésicas descartáveis, de uso odontológico, até o momento, propôs-se, neste trabalho, estudar o grau de padronização das mesmas, comparando-se os resultados com as normas citadas.

\section{MATERIAL E MÉTODO}

Foram avaliadas neste estudo 8 marcas comerciais de agulhas descartáveis para uso odontológico, disponiveis no estado de São Paulo em casas especializadas (Dentais), no segundo semestre de 1997, a saber: BD curta 30 G (BDC) - lote: 0697004; BD longa 27 G (BDL) - lote: 1296003; Ibras curta $30 \mathrm{G}$ (IBC) - lote: 170797L79; Ibras longa 27 G (IBL) - lote: 280497L76; Injecta curta 30 G (INC) - lote: AO196; Misawa curta 30 G (MIC) - lote: 961105; Monoject curta 30 G (MOC) - lote: 242663; Nipro curta 30 G (NIC) - lote: 97D11; Nipro longa $27 \mathrm{G}$ (NIL) - lote: 97D11; Terumo curta 30 G (TEC) - lote: 961226 e Unoject curta 30 G (UNC) - lote: 970604. Para cada teste realizado fo-

\footnotetext{
* Mestre em Ciências; ** Professora Assistente Doutora; *** Professor Titular - Área de Farmacologia, Anestesiologia e Terapêutica da Faculdade de Odontologia de Piracicaba da UNICAMP.
} 
GIROTTO, M. A.; VOLPATO, M. C.; RANALI, J. Avaliação da padronização de agulhas anestésicas odontológicas comercializadas no Brasil. Pesqui Odontol Bras, v. 14, n. 3, p. 294-299, jul./set. 2000.

ram utilizadas 10 agulhas da mesma marca e comprimento, retiradas aleatoriamente a partir da caixa de 100 unidades. Foram avaliados as medidas das agulhas, o grau de corrosão e as informações da caixa e dos invólucros individuais.

\section{Medidas das agulhas}

As medidas de $\mathrm{C}_{1}$ (comprimento da cânula que penetra nos tecidos), $\mathrm{C}_{2}$ (comprimento da cânula que perfura o tubete), $\mathrm{C}_{3}$ (comprimento da cânula no interior do intermediário) e do diâmetro externo foram feitas com paquímetro digital (Mitutoyo Absolute Digimatic - Japan). O "diâmetro interno" foi medido em microscópio de observação (Ernst Leitz Wetzlar - Germany) em aumento de 30 vezes, fixando-se um dos cursores do microscópio de observação em um dos lados da parede interna da agulha, movendo a régua da ocular na direção da parede diametralmente oposta. Para esta medida a agulha era fixada em base.

Após a obtenção das medidas, estas foram comparadas (dentro de cada grupo, agulhas curtas e longas) estatisticamente através do teste de Kruskal-Wallis e em comparações múltiplas, com nível de significância de $5 \%$, pelo programa estatístico JMP versão 3.1 (SAS Institute Inc. ${ }^{12}$, 1995).

\section{Teste de corrosão da cânula}

Este teste foi realizado de acordo com as especificações do ANSI/ADA ${ }^{1}$ - 1986.

\section{Informações na caixa e em seus invólucros individuais}

Para as caixas foi observada a existência das seguintes informações: tamanho e calibre da agulha, número do lote, data de validade, método para a utilização das agulhas e aviso sobre a manutenção da esterilidade da agulha. Os invólucros individuais foram avaliados quanto à existência do selo de união entre as suas duas partes, à visualização nítida do selo, quando violado, às informações sobre o tamanho e calibre da agulha no selo, à data de validade e quanto à padronização de cores entre as marcas analisadas.

\section{RESULTADOS E DISCUSSÃO}

Ao analisar as médias, bem como os valores mínimo e máximo para cada uma das marcas, nos dois grupos estudados, agulhas curtas e longas, pôde-se observar grande variação no comprimento da cânula $\left(\mathrm{C}_{1}\right)$. Para algumas marcas de agulhas curtas como Injecta, Unoject e Misawa esta medida aproxima-se da obtida pela agulha longa da marca BD (Tabelas 1 e 2).

Como a ISO ${ }^{8}$ estabelece como padrão de $\mathrm{C}_{1}$ o valor relatado pelo fabricante, com uma tolerância de $\pm 2 \mathrm{~mm}$, e nenhum dos fabricantes apresenta o comprimento em termos numéricos, mas apenas a classificação em longa ou curta, se considerar-se a média como sendo o valor padrão da empresa, especificamente para as agulhas longas, todas as

TABELA 1 - Média, desvio-padrão, valores mínimo e máximo (em mm) e soma das ordens dos comprimentos de $\mathrm{C}_{1}$, $\mathrm{C}_{2} \mathrm{e}$ $\mathrm{C}_{3}$ e dos diâmetros externo e interno, para as agulhas longas $(27 \mathrm{G})$.

\begin{tabular}{|c|c|c|c|c|c|}
\hline & $\begin{array}{c}\text { Média } \pm \\
\text { D. padrão } \\
C_{1}\end{array}$ & $\begin{array}{c}\text { Média } \pm \\
\text { D. padrão } \\
\mathrm{C}_{2}\end{array}$ & $\begin{array}{c}\text { Média } \pm \\
\text { D. padrão } \\
C_{3}\end{array}$ & $\begin{array}{c}\text { Média } \pm \\
\text { D. padrão } \\
\text { Diâm. externo }\end{array}$ & $\begin{array}{c}\text { Média } \pm \\
\text { D. padrão } \\
\text { Diâm. interno }\end{array}$ \\
\hline $\begin{array}{l}\text { Padrão } \\
\text { ANSI/ADA }(\otimes)\end{array}$ & $34 \pm 4$ & 9,5 a 11 & & 0,39 a 0,42 & 0,19 (no mínimo) \\
\hline Padrão - ISO $(\Delta)$ & $\begin{array}{l} \pm 2 \mathrm{~mm} \text { do valor } \\
\text { estabelecido pelo fabricante }\end{array}$ & 9,0 a 14 & $\geq 5$ & & \\
\hline Padrão NF $(\diamond)$ & & & & $0,40 \pm 0,02$ & 0,19 (no mínimo) \\
\hline $\mathrm{BD}$ & $\begin{array}{c}\text { A } \Delta \\
29,03 \pm 0,71\end{array}$ & $\begin{array}{c}\text { A } \Delta \\
12,26 \pm 0,73\end{array}$ & $\begin{array}{c}\text { A } \Delta \\
7,66 \pm 0,39\end{array}$ & $\begin{array}{l}A \otimes \diamond \\
0,4 \pm 0\end{array}$ & $\begin{array}{c}\text { A } \\
0,19 \pm 0,013\end{array}$ \\
\hline Nipro & $\begin{array}{c}\text { A } \Delta \\
29,25 \pm 0,15\end{array}$ & $\begin{array}{c}\mathrm{B} \Delta \otimes \\
10,77 \pm 0,10\end{array}$ & $\begin{array}{c}\text { B } \Delta \\
9,01 \pm 0,14\end{array}$ & $\begin{array}{c}\mathrm{A} \otimes \diamond \\
0,405 \pm 0,005\end{array}$ & $\begin{array}{c}\text { A } \\
0,19 \pm 0,005 \\
\end{array}$ \\
\hline Ibras & $\begin{array}{c}\mathrm{B} \Delta \otimes \\
34,76 \pm 0,52\end{array}$ & $\begin{array}{c}\mathrm{A} \Delta \\
12,81 \pm 0,29\end{array}$ & $\begin{array}{c}\mathrm{C} \\
5,38 \pm 0,30\end{array}$ & $\begin{array}{c}A \otimes \Delta \\
0,405 \pm 0,005\end{array}$ & $\begin{array}{c}\mathrm{B} \otimes \diamond \\
0,21 \pm 0,009\end{array}$ \\
\hline
\end{tabular}

Médias seguidas por letras distintas (A, B e C) diferem entre si pelo teste de Kruskal-Wallis (p < 0,05); $\mathrm{n}=10$. 
GIROTTO, M. A.; VOLPATO, M. C.; RANALI, J. Avaliação da padronização de agulhas anestésicas odontológicas comercializadas no Brasil. Pesqui Odontol Bras, v. 14, n. 3, p. 294-299, jul./set. 2000.

TABELA 2 - Média, desvio-padrão, valores mínimo e máximo (em mm) e soma das ordens dos comprimentos de $\mathrm{C}_{1}$, $\mathrm{C}_{2}$ e $\mathrm{C}_{3}$ e dos diâmetros externo e interno, para as agulhas curtas (30 G).

\begin{tabular}{|c|c|c|c|c|c|}
\hline & $\begin{array}{c}\text { Média } \pm \\
\text { D. padrão } \\
\mathrm{C}_{1}\end{array}$ & $\begin{array}{c}\text { Média } \pm \\
\text { D. padrão } \\
\mathrm{C}_{2}\end{array}$ & $\begin{array}{c}\text { Média } \pm \\
\text { D. padrão } \\
\mathrm{C}_{3}\end{array}$ & $\begin{array}{l}\text { Média } \pm \\
\text { D. padrão } \\
\text { Diâm. ext. }\end{array}$ & $\begin{array}{l}\text { Média } \pm \\
\text { D. padrão } \\
\text { Diâm. int. }\end{array}$ \\
\hline $\begin{array}{l}\text { Padrão } \\
\text { ANSI/ADA }(\otimes)\end{array}$ & & 9,5 a 11 & & 0,30 a 0,33 & 0,12 (no mínimo) \\
\hline Padrão ISO $(\Delta)$ & $\begin{array}{c} \pm 2 \mathrm{~mm} \text { do valor } \\
\text { estabelecido pelo fabricante }\end{array}$ & & $\geq 5$ & & \\
\hline Padrão NF ( $(\diamond)$ & & & & $0,30 \pm 0,02$ & 0,14 (no mínimo) \\
\hline Monoject & $\begin{array}{c}\mathrm{A} \Delta \\
18,30 \pm 0,24\end{array}$ & $\begin{array}{c}\mathrm{A} \otimes \Delta \\
10,42 \pm 0,17\end{array}$ & $\begin{array}{c}\text { B } \Delta \\
9,46 \pm 0,32\end{array}$ & $\begin{array}{c}\mathrm{B} C \otimes \diamond \\
0,311 \pm 0,003\end{array}$ & $\begin{array}{c}\mathrm{B} \otimes \\
0,14 \pm 0,0082\end{array}$ \\
\hline Ibras & $\begin{array}{c}\text { A B } \Delta \\
19,44 \pm 0,40\end{array}$ & $\begin{array}{c}\text { B C } \Delta \\
12,58 \pm 0,12\end{array}$ & $\begin{array}{c}\mathrm{A} \\
5,01 \pm 0,18\end{array}$ & $\begin{array}{c}A C \otimes \diamond \\
0,305 \pm 0,005\end{array}$ & $\begin{array}{c}\text { A B } \\
0,12 \pm 0,0164\end{array}$ \\
\hline Nipro & $\begin{array}{c}\text { A B } \Delta \\
19,94 \pm 0,18\end{array}$ & $\begin{array}{c}\mathrm{A} \mathrm{D} \otimes \Delta \\
10,53 \pm 0,14\end{array}$ & $\begin{array}{c}\mathrm{B} \Delta \\
9,0 \pm 0,11\end{array}$ & $\begin{array}{l}\mathrm{B} C \otimes \diamond \\
0,31 \pm 0\end{array}$ & $\begin{array}{c}\text { A C } \\
0,12 \pm 0,0038\end{array}$ \\
\hline Terumo & $\begin{array}{c}\text { A B D } \Delta \\
20,62 \pm 0,19\end{array}$ & $\begin{array}{c}\text { A C D } \Delta \\
10,89 \pm 0,13\end{array}$ & $\begin{array}{c}\text { A C } \Delta \\
6,17 \pm 0,29\end{array}$ & $\begin{array}{l}\mathrm{BC} \otimes \diamond \\
0,31 \pm 0\end{array}$ & $\begin{array}{c}\text { B C } \\
0,13 \pm 0,011\end{array}$ \\
\hline $\mathrm{BD}$ & $\begin{array}{c}\text { B C } \\
22,50 \pm 0,71\end{array}$ & $\begin{array}{c}\text { A C D } \Delta \\
10,97 \pm 0,65\end{array}$ & $\begin{array}{c}\text { B C } \Delta \\
7,09 \pm 0,24\end{array}$ & $\begin{array}{c}A \otimes D \\
0,30 \pm 0\end{array}$ & $\begin{array}{c}\text { A B } \\
0,13 \pm 0,0125\end{array}$ \\
\hline Injecta & $\begin{array}{c}\text { C D } \Delta \\
25,36 \pm 0,73\end{array}$ & $\begin{array}{c}\text { A } \Delta \\
10,11 \pm 0,66\end{array}$ & $\begin{array}{c}\text { A } \\
5,39 \pm 0,33\end{array}$ & $\begin{array}{c}A C \otimes \diamond \\
0,305 \pm 0,005\end{array}$ & $\begin{array}{c}\text { B C } \\
0,13 \pm 0,0175\end{array}$ \\
\hline Unoject & $\begin{array}{c}\mathrm{CD} \Delta \\
25,45 \pm 0,22 \\
\end{array}$ & $\begin{array}{c}\mathrm{B} \Delta \\
12,64 \pm 0,13\end{array}$ & $\begin{array}{c}\text { A C } \Delta \\
6,15 \pm 0,17\end{array}$ & $\begin{array}{c}\mathrm{A} \otimes \diamond \\
0,30 \pm 0\end{array}$ & $\begin{array}{c}\text { B C } \\
0,13 \pm 0,0076\end{array}$ \\
\hline Misawa & $\begin{array}{c}\mathrm{C} \Delta \\
25,67 \pm 0,20\end{array}$ & $\begin{array}{c}\text { B D } \Delta \\
11,25 \pm 0,22\end{array}$ & $\begin{array}{c}\text { A C } \Delta \\
6,11 \pm 0,18\end{array}$ & $\begin{array}{c}\mathrm{A} \otimes \diamond \\
0,30 \pm 0\end{array}$ & $\begin{array}{c}\mathrm{A} \\
0,11 \pm 0,0043\end{array}$ \\
\hline
\end{tabular}

Médias seguidas por letras distintas (A, B, C e D) diferem entre si pelo teste de Kruskal-Wallis (p < 0,05); $\mathrm{n}=10$.

marcas atenderiam a essa especificação. Entretanto, se tomar-se por base os valores mínimo e máximo, a marca BD estaria fora dessa especificação. Utilizando-se o mesmo critério para as agulhas curtas, apenas a marca BD não atenderia à especificação da ISO ${ }^{8}$.

Já a norma americana ANSI/ADA ${ }^{1}$ admite variações maiores, de acordo com o tamanho de $\mathrm{C}_{1} \mathrm{e}$, além disso, fixa comprimentos para os diversos tamanhos nominais: extra-curta ( 8 a $14 \mathrm{~mm}$ ), curta $(24 \pm 4 \mathrm{~mm})$, longa $(34 \pm 4 \mathrm{~mm})$ e extra-longa $(41 \pm 4 \mathrm{~mm})$. Nesse aspecto, essa norma é mais criteriosa e completa, pois leva em conta a finalidade (tipo de bloqueio) à qual se destina cada um dos tamanhos, apesar da grande variação expressa entre os valores mínimos e máximos. Embora não faça parte da norma, a American Dental Association faz recomendações específicas a respeito do calibre ideal para cada tipo de anestesia. Assim, as agulhas extra-curtas $(30 \mathrm{G})$ seriam indicadas para injeções intraligamentares, intra-ósseas, intrapulpares e injeções no palato, enquanto as técnicas infiltrativas deveriam ser feitas com agulhas curtas de calibre 23, 25 ou 27 G. Já para os bloqueios regionais, que requerem penetração mais profunda, o ideal seria utilizar agulhas longas ou extra-longas, com calibres 23 ou 25 G. Para técnicas com baixa incidência de aspiração positiva, como os bloqueios dos nervos palatino maior, nasopalatino, incisivo e mentoniano, alguns autores como MALAMED ${ }^{9}$ (1997), colocam como opção o uso de agulhas curtas $27 \mathrm{G}$.

Considerando-se a norma americana, para as agulhas longas, as marcas BD e Nipro apresentaram-se fora da especificação (34 $\pm 4 \mathrm{~mm}$ ). Estes dados são importantes, porque ao utilizar agulhas 
GIROTTO, M. A.; VOLPATO, M. C.; RANALI, J. Avaliação da padronização de agulhas anestésicas odontológicas comercializadas no Brasil. Pesqui Odontol Bras, v. 14, n. 3, p. 294-299, jul./set. 2000.

com comprimento menor, como é o caso das marcas citadas, ou o profissional não chega à profundidade adequada (deixando $5 \mathrm{~mm}$ da cânula para fora dos tecidos, como prevenção no caso de fratura da agulha), não obtendo anestesia satisfatória, ou então introduz a agulha até o intermediário.

A importância destes dados torna-se ainda maior, quando são avaliados os resultados de comprimento de $\mathrm{C}_{1}$ para as agulhas curtas. Considerando-se o intervalo admitido pela ANSI/ADA (20 a $28 \mathrm{~mm}$ ), as marcas Terumo, BD, Injecta, Unoject e Misawa estariam corretas. Entretanto, esta mesma norma estabelece que as agulhas curtas devem ter um calibre mínimo de $27 \mathrm{G}$ (diâmetro externo variando entre $0,39 \mathrm{~mm}$ a $0,42 \mathrm{~mm}$ ), o que não acontece com as marcas comercializadas no Brasil, pois todas apresentam calibre $30 \mathrm{G}$. Esta exigência baseia-se no fato de que algumas das indicações destas agulhas envolvem bloqueios, nos quais é necessária a realização de aspiração, que se torna mais dificil com as agulhas de menor calibre, como no caso da agulha $30 \mathrm{G}^{5,13}$. Além disso, como observado nos trabalhos de HAMBURG $^{7}$ (1972), FULLER et al. ${ }^{6}$ (1979), MOLLEN et al. ${ }^{10}$, (1981) e BROWNBILL et $a l^{4}{ }^{4}$ (1987), o paciente não consegue diferenciar, através da sensação dolorosa, a punção por agulhas de diversos calibres, não se justificando o uso destas agulhas.

$\mathrm{O}$ comprimento de $\mathrm{C}_{2}$, que garante a introdução da agulha no tubete, é normatizado pela ISO $(9,0$ a $14 \mathrm{~mm}$ ) e ANSI/ADA (9,5 a $11 \mathrm{~mm})$. Se $\mathrm{C}_{2}$ for muito curto, a agulha poderá sair do tubete quando executada a manobra de aspiração, ou até mesmo, não conseguir perfurar seu diafragma, inviabilizando assim a realização da anestesia, como observado por PIESOLD et al. ${ }^{11}$ (1998) ao avaliarem algumas marcas de agulhas. Quando $\mathrm{C}_{2}$ é muito longo, parte da solução anestésica permanecerá no tubete, não sendo possivel utilizar todo seu conteúdo. No presente trabalho todas as agulhas enquadraram-se na norma da ISO (Tabelas 1 e 2) e apenas as agulhas Monoject curta e Nipro longa e curta estavam de acordo com a norma ANSI/ADA (com intervalo mais restrito para permitir o uso de todo conteúdo do tubete).

Somente a ISO ${ }^{8}$ estabelece um valor para $\mathrm{C}_{3}$ de pelo menos $5 \mathrm{~mm}$ (Tabelas 1 e 2). No presente trabalho, apenas duas marcas (Injecta curta e Ibras longa e curta) ficaram aquém deste padrão. O diâmetro externo foi a medida que apresentou menor variação em todas as marcas e tamanhos, estando todas de acordo com as normas ANSI/ADA e Francesa. O diâmetro interno, porém, mostrou variação maior (Tabelas 1 e 2). Apesar dessa variação, não foram encontradas aberrações, como no trabalho de WITTROCK; FISCHER ${ }^{14}$ (1968).

Com relação à integridade do metal, na forma preconizada pela ANSI/ADA (observação a olho nu), todas as agulhas analisadas no estudo de corrosão preencheram as especificações do teste, tendo sido observada apenas uma discreta perda de brilho de algumas amostras das marcas Injecta, Ibras e Monoject, porém sem a visualização de pontos de corrosão. Embora não especificado no teste, a observação das agulhas ao microscópio óptico (aumento de $30 \mathrm{X}$ ) revelou a existência de pontos de corrosão imperceptiveis a olho nu, tanto em áreas com perda de brilho quanto em áreas normais. Foi observada ainda falta de padronização no acabamento do bisel de $\mathrm{C}_{1}$ (um lado diferente do outro, presença de farpas e material plástico obstruindo o lúmen e bisel com formato de anzol), além de agulhas com o bisel perfurando o invólucro plástico e apresentando angulação diferente de $90^{\circ}$ em relação ao intermediário.

Para um material utilizado para introdução de substâncias na intimidade dos tecidos, essas irregularidades não deveriam ocorrer. É interessante notar que, apesar de existir especificação para o tipo de aço a ser utilizado neste material, tanto na Norma Americana, quanto na Francesa e na ISO, somente a primeira estabelece um teste específico para o produto acabado (a agulha).

Além dessas deficiências, foi observado ainda, neste trabalho, que algumas marcas não apresentavam as informações de data de fabricação e validade com clareza, havendo ainda uma marca importada que não apresentava data de validade, ferindo o Código de Defesa do Consumidor.

As marcas Misawa e Monoject (ambas importadas), não apresentavam selo, mas apenas uma marca feita com metal aquecido, para unir as duas partes do protetor da agulha, tornando dessa forma, dificil observar se os invólucros já haviam sido abertos ou não. Da mesma forma, as marcas Terumo e Unoject e, de maneira mais evidente, a marca Nipro também não permitiam a visualização do rompimento do lacre, quando violado, devido a possuírem um picotamento muito regular no sentido horizontal. Já as marcas BD, Ibras e Injecta, com picotamento no sentido transversal, permitiam a rápida visualização da violação do selo.

Com relação ao invólucro protetor, existe uma variação de cores para os diferentes tipos de marcas e tamanhos, de acordo com a deliberação do próprio fabricante, exigindo do usuário maior atenção, embora as agulhas longas tenham o protetor ou o lacre sempre nas cores laranja, branca 
GIROTTO, M. A.; VOLPATO, M. C.; RANALI, J. Avaliação da padronização de agulhas anestésicas odontológicas comercializadas no Brasil. Pesqui Odontol Bras, v. 14, n. 3, p. 294-299, jul./set. 2000.

TABELA 3 - Informações contidas na caixa e no invólucro individual, para as agulhas longas e curtas.

\begin{tabular}{|c|c|c|c|c|c|c|c|c|c|c|}
\hline & & \multicolumn{3}{|c|}{ Data } & \multirow{2}{*}{$\begin{array}{c}\text { Método de } \\
\text { esterilização }\end{array}$} & \multicolumn{2}{|c|}{$\begin{array}{l}\text { Informações } \\
\text { necessárias }\end{array}$} & \multirow{2}{*}{$\begin{array}{c}\text { Visualização } \\
\text { da violação } \\
\text { do lacre }\end{array}$} & \multicolumn{2}{|c|}{ Cor } \\
\hline & & Fabricação & Validade & Lote & & Caixa & Lacre & & Invólucro & Lacre \\
\hline \multirow[t]{2}{*}{$\mathrm{BD}$} & Curta & \multirow{2}{*}{ Sim } & \multirow{2}{*}{ Sim } & \multirow{2}{*}{ Sim } & \multirow{2}{*}{$\begin{array}{l}\text { Óxido de } \\
\text { etileno }\end{array}$} & \multirow{2}{*}{ Sim } & \multirow{2}{*}{ Sim } & \multirow{2}{*}{ Sim } & $\mathrm{B}$ & $\mathrm{B}$ e $\mathrm{V}$ \\
\hline & Longa & & & & & & & & $\mathrm{B}$ & $\mathrm{B}$ e $\mathrm{L}$ \\
\hline \multirow[t]{2}{*}{ Ibras } & Curta & \multirow{2}{*}{ Sim* } & \multirow{2}{*}{$\operatorname{Sim} *$} & \multirow{2}{*}{$\operatorname{Sim} *$} & \multirow{2}{*}{$\begin{array}{c}\text { Raios gama e } \\
\text { cobalto } 60\end{array}$} & \multirow{2}{*}{ Sim } & \multirow{2}{*}{ Sim } & \multirow{2}{*}{ Sim } & $\mathrm{V}$ & $\mathrm{B}$ \\
\hline & Longa & & & & & & & & $\mathrm{L}$ & $\mathrm{B}$ \\
\hline \multicolumn{2}{|c|}{ Injecta } & Sim & $\operatorname{Sim}$ & $\operatorname{Sim}$ & Raios gama** & Não & Não & Sim & $\mathrm{B}$ & $\mathrm{V}$ \\
\hline \multicolumn{2}{|c|}{ Misawa } & $\operatorname{Sim}$ & $\operatorname{Sim}$ & Sim & Óxido de etileno & Sim & Sem lacre & Não & $\mathrm{T}$ & sem selo \\
\hline \multicolumn{2}{|c|}{ Monoject } & Não & Não & Sim & Não divulga & Não & Sem lacre & Não & A & sem selo \\
\hline \multirow{2}{*}{ Nipro } & Curta & \multirow{2}{*}{ Não } & \multirow{2}{*}{ Sim } & \multirow{2}{*}{ Sim } & \multirow{2}{*}{$\begin{array}{l}\text { Óxido de } \\
\text { etileno** }\end{array}$} & \multirow{2}{*}{ Não } & \multirow{2}{*}{ Não } & \multirow{2}{*}{ Não } & $\mathrm{T}$ & A \\
\hline & Longa & & & & & & & & $\mathrm{T}$ & $\mathrm{L}$ \\
\hline \multicolumn{2}{|c|}{ Terumo } & Sim* & Sim & Sim & Óxido de etileno & Sim & Não & Não & $\mathrm{B}$ & $\mathrm{B}$ e $\mathrm{V}$ \\
\hline \multicolumn{2}{|c|}{ Unoject } & Sim* & Sim & Sim & Não divulga & Não & Não & Não & $\mathrm{B}$ & $\mathrm{A}$ e B \\
\hline
\end{tabular}

* Falta clareza às informações. ** Falta do aviso de manutenção de esterilidade. Cor estabelecida pelo fabricante no invólucro e no lacre (em siglas): $\mathrm{B}=$ branca; $\mathrm{V}=$ verde; $\mathrm{L}=$ laranja; $\mathrm{T}$ = transparente; $\mathrm{A}=$ azul.

ou uma combinação dessas cores. Apesar dessa especificação só existir na Norma Francesa, sua aplicação parece ser relativamente simples, já existindo essa padronização pela $\mathrm{ABNT}^{2}$ (1997) das agulhas hipodérmicas para seringa tipo Luer (Tabela 3).

\section{CONCLUSÃO}

Frente ao exposto, conclui-se que há necessidade de se estabelecer uma norma técnica nacional, para fabricação de agulhas anestésicas descartáveis para uso odontológico no Brasil. Neste sentido, espera-se que este estudo possa contribuir para uma discussão mais ampla do assunto, pos- sibilitando a determinação de uma norma que reflita as necessidades de seus usuários, dentistas e pacientes.

\section{AGRADECIMENTOS}

À Área de Materiais Dentários da FOP/UNICAMP pela cessão do laboratório e aparelhos utilizados para a medida das agulhas, bem como ao técnico deste, Engenheiro Marcos Antonio Cangiani.

Ao Conselho Nacional de Desenvolvimento Científico e Tecnológico (CNPq), pela concessão de bolsa de Mestrado ao autor Marcos Antonio Girotto (processo $\left.\mathrm{n}^{\circ} 136992 / 96-0\right)$.

GIROTTO, M. A.; VOLPATO, M. C.; RANALI, J. Evaluation of the standardization of injection dental needles marketed in Brazil. Pesqui Odontol Bras, v. 14, n. 3, p. 294-299, jul./set. 2000.

The standardization of sterile, single-use dental injection needles marketed in Brazil in 1997 was evaluated. The effective needle length (L1), butt end length (L2), socket depth (L3), external (ED) and internal (ID) diameters, corrosion resistance of the cannula, and the information printed on the package (unitary protector and box) were evaluated. The data were compared with the International Standard (ISO), American specification no. 54 (ANSI/ADA) and French Standard (FS). There were great variations for L1, L2, L3 and ID, and no trade mark fitted in any of the standards for all the dimensions. The external diameter showed very little variation in all the trade marks according to the ANSI/ADA and FS. All of the needles withstood the corrosion test. Some trade marks had incomplete information in their package and one of them had no expiration date. Some of the unitary protectors, once opened, did not show clear evidence of having been violated. These results show the need for a Brazilian specification to regulate the manufacturing of single-use dental injection needles.

UNITERMS: Needles; Quality control. 
GIROTTO, M. A.; VOLPATO, M. C.; RANALI, J. Avaliação da padronização de agulhas anestésicas odontológicas comercializadas no Brasil. Pesqui Odontol Bras, v. 14, n. 3, p. 294-299, jul./set. 2000.

\section{REFERÊNCIAS BIBLIOGRÁFICAS}

1. AMERICAN STANDARDS INSTITUTE, New York. ANSI/ADA Specification $\mathbf{n}^{\circ} \mathbf{5} 4$ For double-pointed, parenteral, single-use needles for Dentistry. New York, Sept. 1986. 9 p.

2. ASSOCIAÇÃO BRASILEIRA DE NORMAS TÉCNICAS, Rio de Janeiro. NBR 9259: Agulha hipodérmica estéril e de uso único. Rio de Janeiro, 1997. 9 p.

3. BRASIL. Lei $n^{\circ} 8.078$. Dispõe sobre a proteção do consumidor e dá outras providências. Diário Oficial da União, Brasília, p. 12, 11 de set. de 1990.

4. BROWNBILL, J. W.; WALKER, P. O.; BOURCY, B. D. et al. Comparison of inferior dental nerve block injections in child patients using 30-gauge and 25-gauge short needles. Anesth Prog, v. 34, p. 215-216, Feb. 1987.

5. COOLEY, R. C.; ROBISON, S. Comparative evaluation of the 30-gauge dental needle. Oral Surg Oral Med Oral Pathol, v. 48, n. 5, p. 400-404, 1979.

6. FULLER, N. P.; MENKE, R. A.; MEYERS, W. J. Perception of pain to three different intraoral penetrations of needles. J Am Dent Assoc, v. 99, p. 822-824, 1979.
7. HAMBURG, H. L. Preliminary study of patient reaction to needle gauge. N Y State Dent J, v. 38, n. 7, p. 425-426, Apr./Sept. 1972.

8. INTERNATIONAL ORGANIZATION FOR STANDARDIZATION, Genova. ISO 7885: Sterile, single-use dental injection needle. Genova, 1996. 5 p.

9. MALAMED, S. F. Handbook of local anesthesia. 4. ed. St. Louis: Mosby, 1997. 327 p.

10. MOLlEN, A. J.; FICARA, A. J.; PROVANT, D. R. Needles 25 gauge versus 27 gauge - can patients really tell? Gen Dent, v. 29, p. 417-418. Sept./Oct. 1981.

11. PIESOLD, J.; MÜLLER, W.; DREIBIG, J. An experimental study on the aspirating reliability of different types of injection syringes with regard to the formation of punch cylinders. Br J Oral Maxillofac Surg, v. 36, p. 39-43, 1998.

12. SAS Institute Inc. (ed): JMP ${ }^{\circledR}$ versão 3.1. User's guide: version 3.1 of JMP. Cary : SAS Institute, 1995.

13. SMITH, N. An investigation of the influence of gauge on some physical properties of hypodermic needles. Aust Dent J, v. 13, p. 158-163, Apr. 1968.

14. WITTROCK, J. W.; FISCHER, W. E. The aspiration of blood through small-gauge needles. J Am Dent Assoc, v. 76, p. 79-81, Jan. 1968.

Recebido para publicação em 11/08/99

Enviado para reformulação em 31/03/00

Aceito para publicação em 10/05/00 